\title{
Sentimental Analysis in Social Media using IGBA Algorithm
}

\author{
M. Yuvaraja \\ Assistant Professor, \\ Department of Computer Science, \\ Bishop Ambrose College, \\ Coimbatore, Tamil Nadu, India
}

\author{
S. Thavamani, $\mathrm{PhD}$ \\ Associate Professor, \\ Department of Computer Applications, \\ Sri Ramakrishna College for Arts and Science, \\ Coimbatore, Tamil Nadu, India
}

\begin{abstract}
Social media observance has been growing day by day therefore analysing of social information plays a very important role in knowing user behaviour. This system has a tendency to square measure analysing Social knowledge like Twitter Tweets victimization sentiment analysis that checks the perspective of User post and review. This paper develops a new algorithm improved gradient boost algorithm is combined lexicon supported social media keywords and on-line review, post and conjointly realize hidden relationship pattern from these keyword. Finally proposed novel algorithm IGBA provide better performance compared with existing algorithm naïve Bayes classifier, Support vector machine classifier.
\end{abstract}

\section{Keywords}

Data Mining, Sentiment Analysis, Social Network, Support Vector Machine, Naïve Bayes

\section{INTRODUCTION}

An increasing variety of individuals is increasingly approaching to the social networking sites, that become a lot of and a lot of common and complex at common their context several and varied communities area unit originated by users with common interests or with similar ways in which to feel a part of the community[5]. Social media is changing into a vital topic for research in several fields. As variety of individual's using social network are growing day by day, to speak with their peers in order that they'll share their personal feeling every day and views are created on massive scale.

Social Media observance or trailing is most vital topic in today's current situation. In nowadays several firms are using social media selling to advertise their merchandise or brands, thus it becomes essential for them that they'll be ready to calculate the success and utility of every product [6]. Sentiment analysis is that the Natural Language Processing (NLP) task handling the detection and classification of sentiments in texts.

Usually, the categories thought of "positive", "negative" and "neutral", though in some cases finergrained classes are inserted (e.g. "very positive" and "very negative") or solely the "positive" and "negative" categories are taken under consideration. Another connected task are feeling detection, considerations the classification of text into many categories of feeling.

\section{RELATED WORKS}

H. Becker et al [4] have proposed work that tries to analyse and interpret the public sentiment variations in micro blogging services. Two novel generative models are developed to solve the reason mining problem. The two proposed models are general: they can be applied to other tasks such as finding topic differences between two sets of documents. We propose a sentimental data analysis model using Neural Networks. Both positive and negative feed backs will be calculated here. These foreground topics can give potential interpretations of the sentiment variations. To further enhance the readability of the mined reasons, we select the most representative tweets for foreground topics and develop another generative model called Reason Candidate and Background LDA (RCB-LDA) to rank them with respect to their popularity within the variation period.

J. Bollen et al [2] has developed to perform a sentiment analysis of all tweets published on the micro blogging platform Twitter in the second half of 2008. We use a psychometric instrument to extract six mood states (tension, depression, anger, vigour, fatigue, confusion) from the aggregated Twitter content and compute a sixdimensional mood vector for each day in the timeline. We compare our results to a record of popular events gathered from media and sources. We find that events in the social, political, cultural and economic sphere do have a significant, immediate and highly specific effect on the various dimensions of public mood. We speculate that large scale analyses of mood can provide a solid platform to model collective emotive trends in terms of their predictive value with regards to existing social as well as economic indicators.

G. Heinrich [3] has proposed Presents parameter estimation methods common with discrete probability distributions, which is of particular interest in text modelling. Starting with maximum likelihood, a posteriori and Bayesian estimation, central concepts like conjugate distributions and Bayesian networks are reviewed. As an application, the model of latent Dirichlet allocation (LDA) is explained in detail with a full derivation of an approximate inference algorithm based on Gibbs sampling, including a discussion of Dirichlet hyper parameter estimation.

B.Pangand et al [1] has developed a survey covers techniques and approaches that promise to directly enable opinion-oriented information-seeking systems. Our focus is on methods that seek to address the new challenges raised by sentiment-aware applications, as compared to those that are already present in more traditional fact-based analysis. We include material on summarization of evaluative text and on broader issues regarding privacy, manipulation, and economic impact that the development of opinion-oriented information-access services gives rise to. To facilitate future work, a discussion of available 
resources, benchmark datasets, and evaluation campaigns is also provided.

Paolo Fornacciari et al [5] this paper presents a possible combined approach between Social Network Analysis and Sentiment Analysis. In particular, we have tried to associate a sentiment to the nodes of the graphs showing the social connections, and this may highlight the potential correlations. The idea behind it is that, on the one hand, the network topology can contextualize and then, in part, unmask some incorrect results of the Sentiment Analysis; on the other hand, the polarity of the feeling on the network can highlight the role of semantic connections in the hierarchy of the communities that are present in the network. In this work, we illustrate the approach to the issue, together with the system architecture and, then, we discuss our first results.

Rajni Singh et al [6] has develops a combined dictionary based on social media keywords and online review and also find hidden relationship pattern from these keyword. Social media Monitoring has been growing very rapidly so there is a need for various organizations to analyse customer behaviour or attitude of particular product or any movie review. So, the concepts of sentiment analysis have been introduced. Text analytics and sentiment analysis can help organization to derive valuable business insights. Attitude can be calculated based on polarity check. Sentiment analysis on Online review are done by forming dictionary which shows that it is easier to build dictionary on phrases but complex in case of Twitter as tweets consist of short hands as online review were written in more clear way as compared to Tweets. So, form hidden relationship between different keywords and a dictionary of the words on the basis of different categories of comments \& tweets.

T.-K.Fanand et al [7] Web advertising (Online advertising), a type of advertising that uses the globe Wide internet to draw in customers, has become one in every of the world's most significant promoting channels. This paper addresses the mechanism of Content headed advertising (Contextual advertising), that refers to the assignment of relevant ads inside the content of a generic web content, e.g. blogs. As blogs become a platform for expressing personal opinion, they naturally contain numerous sorts of expressions, as well as each fact and comment of each a positive and negative nature. During this thesis, we tend to propose the employment of sentiment detection to enhance Web-based discourse advertising. The planned SOCA (Sentiment-Oriented discourse Advertising) framework aims to mix discourse advertising matching with sentiment analysis to pick out ads that area unit associated with the positive (and neutral) aspects of a journal and rank them in keeping with their connexion.

Y.Lu et al [8] has develop As Web 2.0 applications become progressively in style, additional and additional individuals categorical their opinions on the net in varied ways in which in real time. Such wide coverage of topics and abundance of users build the net an especially valuable supply for mining people's opinions concerning all types of topics. This system proposing novel and general process techniques for 3 synergistic tasks: (1) group action relevant opinions from all types of internet two.0 sources and organizing them on totally \{different completely different aspects of the subject that not solely is a linguistics grouping of opinions however additionally facilitates user navigation into the large opinion space; (2) inferring the feelings within the opinions with regard to different aspects and different opinion holders, thus on offer the users with a additional careful and wise to multiperspective read of the opinions; and (3) rising the prediction of opinion quality that critically decides the utility of the knowledge extracted from the opinions.

Pooja Khanna et al [9] has developed a system for identify the sentiment in opinion mining from the twitter data. This system implemented by using of R tool. Finally this system retrieved people have used more positive words as compared to negative words.

G. Parthasarathy et al [10] has described the review of sentimental analysis of journal citation. This system produces the frequent citation in multiple research articles and analysis of techniques, methods in sentiment analysis of citation journal. This survey is through the study of the sentiment analysis difficult in journal citation to identify different trends and recommend the forthcoming research directions

Surya Prakash Sharma et al [11] have described review of sentimental analysis of on customer review documents. This paper study on opinion mining in different techniques used, architecture and tools used and challenges.

Akshi Kumar et al[12] has proposed hybrid approach using together corpus based and dictionary based methods to find the semantic coordination of the opinion words in tweets.

Xing Fang et al [13] has proposed a system based on product review data to analyse the categories of sentiment. The product reviews collected from the amazon site and apply the SVM techniques to determine the sentiment analysis of positive, negative and neutral.

Brian Keith et al [14] have developed a system for analysis of sentiment in conference paper reviews using hybrid algorithm. This system to evaluate the capability and performance of the proposed approaches relative to a baseline, using standard metrics, such as accuracy, precision, recall, and the F1-score.

\section{PROPOSED METHODOLOGY}

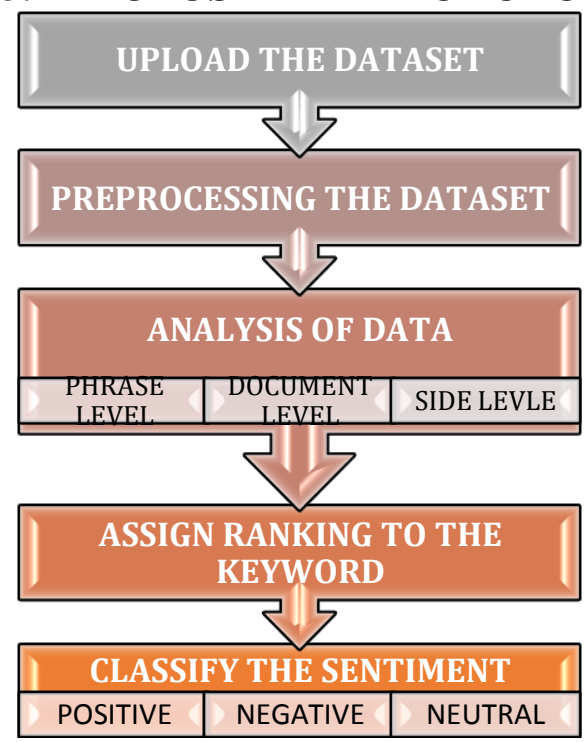

Figure 1: work Flow of Sentiment Analysis 


\subsection{Improved Gradient Boost Algorithm}

One will indiscriminately specify each the loss performs and also the base learner models on demand. In observe, given some specific loss perform (y, f) and/or a custom base-learner $h(x, \theta)$, the answer to the parameter estimates is troublesome to get. To alter this, it absolutely was projected to settle on a brand new perform $\mathrm{h}(\mathrm{x}, \theta \mathrm{t})$ to be the foremost parallel to the negative gradient $\mathrm{N} \mathrm{i}=$ one on the determined data:

$$
g_{f}(x)=E_{y}\left[\frac{\partial \Psi(y, f(x))}{\partial f(x)} \mid x\right]_{f(x)=\hat{f}^{-1}(x)}
$$

Instead of craving for the final resolution for the boost increment within the perform house, one will merely select the new perform increment to be the foremost correlate with $-\operatorname{gt}(\mathrm{x})$. This allows the replacement of a doubtless terribly arduous optimisation task with the classic leastsquares reduction one:

$$
\left(\rho_{t}, \theta_{t}\right)=\arg \min _{\rho, \theta} \sum_{i=1}^{N}\left[-g_{t}\left(x_{i}\right)+\rho h\left(x_{i}, \theta\right)\right]^{2}
$$

To summarize, we are able to formulate the whole sort of the gradient boosting formula, as originally projected by economist. The precise sort of the derived formula with all the corresponding formulas can heavily rely on the planning selections of $(\mathrm{y}, \mathrm{f})$ and $\mathrm{h}(\mathrm{x}, \theta)$. One will realize some common samples of this formula.

- Input data (x, y) $\mathrm{N}$ i=1

- Number of iterations M

- $\quad$ Choice of the loss-function (y, f)

- $\quad$ Choice of the base-learner model $h(x, \theta)$

\section{Algorithm:}

1. initialize f0 with a constant

2. $\quad$ for $\mathrm{t}=1$ to $\mathrm{M}$ do

3. compute the negative gradient $\mathrm{gt}(\mathrm{x})$

4. fit a new base-learner function $\mathrm{h}(\mathrm{x}, \theta \mathrm{t})$

5. find the best gradient descent step-size $\rho t: \rho t=\arg$ $\min \rho \mathrm{N}$ i=1 yi, ft-1 (xi) $+\rho h(x i, \theta t)$

6. update the function estimate: $\mathrm{ft} \leftarrow \mathrm{ft}-1+\rho \mathrm{th}(\mathrm{x}, \theta \mathrm{t})$

7. end for

\subsection{Models in Sentimental Analysis:}

Different categories of Sentiment Analysis There square measure 3 categories of sentiments .i.e. positive, negative and neutral sentiments.

\subsubsection{Positive Sentiments}

This refers to positive angle of the speaker concerning the text. Emotions with positive sentiments replicate happiness, joy, smile etc. just in case of political reviews, if the positive reviews/sentiments concerning the politician square measure a lot of, it suggests that individuals square measure pleased with his work.

\subsubsection{Negative Sentiments}

This refers to negative angle of the speaker concerning the text. Emotions with negative sentiments replicate disappointment, jealousy, hate etc. just in case of political reviews, if the negative reviews/sentiments concerning the politician square measure a lot of, it suggests that individuals aren't pleased with his work.

\subsubsection{Neutral Sentiments}

Here no emotions square measure mirrored concerning the text. It's neither most well-liked nor neglected. Though this category doesn't imply something, it's important for higher distinction between positive and negative categories.

\subsection{Levels of Sentiment Classification}

There square measure 3 sentiment classification levels. I.e. phrase level, document level and side level sentiment classification.

\subsubsection{Phrase Level Classification}

Phrase refers to combination of 2 or over 2 words. Phrase is taken is thought and sentiments square measure classified consequently. However it generally provides inaccurate results owing to addition of negation word. It 1 st determines whether or not the phrase is neutral or polar. It's polar then classified into positive and negative categories.

\subsubsection{Document Level Classification}

It takes into thought one topic and classifies sentiment as positive, negative, or neutral. Generally it's not helpful once there comparison between 2 product that have similar options.

\subsubsection{Side Level Classification}

It determines whether or not the expressed opinion concerning the side or feature is positive, negative or neutral. Here side refers to the element of the entity. This classification level yields terribly fine grained opinion data which may be helpful for varied domains in sentiment analysis. The general opinion is related to the feature of the entity. For example the entity perhaps apple iphone and it has side perhaps with battery, camera, and screen.

The performance of sentiment classification can be evaluated by using four indexes calculated as the following equations:

Accuracy $=(\mathrm{TP}+\mathrm{TN}) /(\mathrm{TP}+\mathrm{TN}+\mathrm{FP}+\mathrm{FN})$

Precision $=\mathrm{TP} /(\mathrm{TP}+\mathrm{FP})$

Recall $=\mathrm{TP} /(\mathrm{TP}+\mathrm{FN})$

$\mathrm{F} 1=(2 \times$ Precision $\times$ Recall $) /($ Precision Recall $)$

In which $\mathrm{TP}, \mathrm{FN}, \mathrm{FP}$ and $\mathrm{TN}$ refer respectively to the number of true positive instances, the number of false negative instances, the number of false positive instances and the number of true negative instances, as defined.

All the problems, requirement and drawbacks are overcome in the proposed system, through developing a dedicated sentimental application platform for this analysis model. This sentimental application will make a major impact and vital program among the market data analysts and researchers like data analyst, data reporter, business analyst, business growth predictors and etc. This 
sentimental application is easy, simple and user friendly to use all types of commercial analysis model. Here raw data will be given as the input and out the output will a tremendous data reporting model.

\section{EXPERIMENT RESULT AND EVALUATION}

Original tweets with labelled sentiments. These halftracked sentiments are positive, negative and neutral categories. For the task of sentiment analysis got and used Support Vector Machine, improve gradient boosting rule that estimates the strength of positive and negative sentiment briefly texts, even for casual language sentiments area unit classified on the idea of adjectives. Even large integer the prevailing system touch upon large information, the getting results area unit terribly less. No differing kinds of results area unit made within the existing system. According to the sentimental analysis, there are a unit numerous sentiments area unit accessible like positive, negative and neutral. Within the existing system, the system categorizes solely the most important positive and negative classes solely. It was not finding exhaustive for the information analysis. The most important classes won't provide a refined result and clarity info from the information set. Conjointly within the existing system major techniques don't seem to be enforced. The table 1 represent the categories of text in the comment in tweeter.

Table 1: Categories of Words

\begin{tabular}{|c|c|}
\hline Positive Category & Negative Category \\
\hline + This product is so good & $\begin{array}{l}\text { - I hate that product } \\
\text { after delivery }\end{array}$ \\
\hline $\begin{array}{l}\text { + I am Happy to get such } \\
\text { a good product }\end{array}$ & $\begin{array}{l}\text { - I won't buy this } \\
\text { product any more }\end{array}$ \\
\hline
\end{tabular}

While comparing with the existing system, the proposed system has been improved a lot by adding more techniques. The table 2 represent the classification of sentiments in text dataset.

Table 2: Classify the Sentiment in Text

\begin{tabular}{|c|c|c|c|}
\hline Data Set & Positive & Negative & Neutral \\
\hline $\begin{array}{l}\text { This product is more excellent } \\
\text { and good }\end{array}$ & Yes & No & No \\
\hline $\begin{array}{l}\text { I am more disappointed in this } \\
\text { product }\end{array}$ & No & Yes & No \\
\hline $\begin{array}{l}\text { I am more disappointed in this } \\
\text { product. I won't buy this } \\
\text { anymore }\end{array}$ & No & Yes & No \\
\hline I am ok with this product & Yes & No & Ok \\
\hline $\begin{array}{l}\text { This is not worthy for } 1000 \\
\text { rupees }\end{array}$ & No & Not & No \\
\hline $\begin{array}{l}\text { After buying the mobile phone, } \\
\text { there are many scratches in the } \\
\text { panel. }\end{array}$ & No & No & $\begin{array}{l}\text { No sentimental words found. } \\
\text { Scratches are the common } \\
\text { word. }\end{array}$ \\
\hline
\end{tabular}

The below Table 3 shows the dataset space of the existing algorithm and proposed algorithm is produce the sentiment classification in word.

Table 3: Different Algorithm Perform Sentiment Classification

\begin{tabular}{|l|c|c|c|c|c|}
\hline S.No & Algorithm & Data & Positive & Negative & Neutral \\
\hline 1 & NB & 190 & 100 & 30 & 60 \\
\hline 2 & SVM & 190 & 105 & 25 & 60 \\
\hline 3 & IGBA & 190 & 120 & 20 & 50 \\
\hline
\end{tabular}




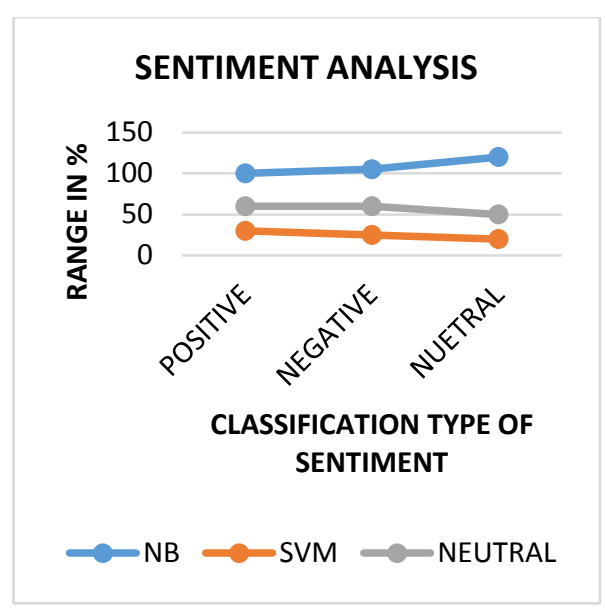

Figure 2: classification of sentiment Analysis in text

The Figure 2 shows the classification of sentiment Analysis in text using NB, SVM and proposed algorithm IGBA. The table 4 shows the accuracy of the existing algorithm and proposed algorithm.

Table 4: Accuracy for Different Algorithm

\begin{tabular}{|c|c|c|}
\hline S.No & Algorithm & Accuracy \\
\hline 1 & NB & 89.53 \\
\hline 2 & SVM & 93.5 \\
\hline 3 & IGBA & 97.4 \\
\hline
\end{tabular}

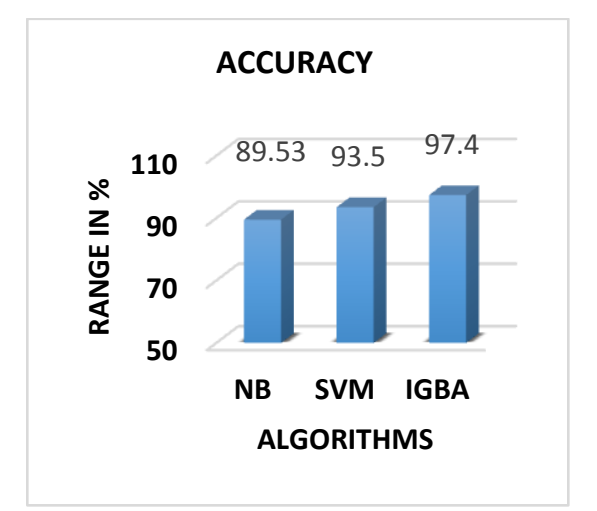

Figure 3: comparing performance of different Algorithm

The Figure 3: comparing performance of different Algorithm and in diagrammatic way represent high performance in proposed algorithm. The Table 5 shows the precision and recall value comparison of existing and proposed algorithm.

Table 6: Precision and Recall value

\begin{tabular}{|c|c|c|}
\hline Algorithm & Precision & Recall \\
\hline NB & 86 & 73 \\
\hline SVM & 87 & 76 \\
\hline IGBA & 89 & 70 \\
\hline
\end{tabular}

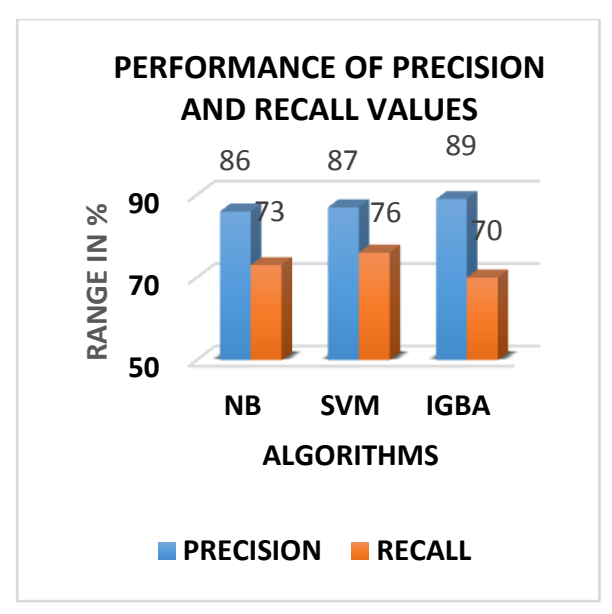

Figure 5: comparison of Precision and Recall value

From the figure 5 and table 6 it shows the performance measure based on the efficiency and the precision compassion of existing (NB, SVM) and proposed (IGBA). The below Table shows the time period for data retrieval comparison of existing and proposed algorithm

Table 7: comparison of Time

\begin{tabular}{|c|c|c|}
\hline Algorithm & Data & Time Period \\
\hline NB & 190 & $3.64(\mathrm{sec})$ \\
\hline SVM & 190 & $3.13(\mathrm{sec})$ \\
\hline IGBA & 190 & $2.63(\mathrm{sec})$ \\
\hline
\end{tabular}

From the figure 6 and table 7 it shows the performance measure based on the retrieval time and the proposed approach IGBA yields better results than the existing (NB SVM).

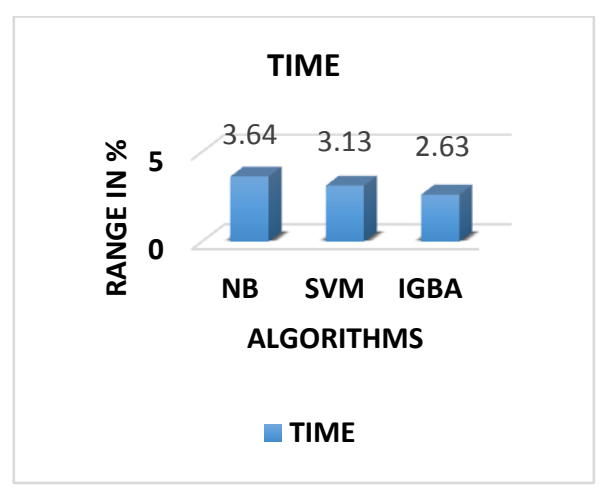

Figure 6: Comparison of Time

\section{CONCLUSION AND FUTURE WORK}

Social media is a fast growing technology in day to day rapidly then they need to analysis the customer behaviour in particular product or any movies review or tweeter, Facebook reviews. This system uses the tweeter dataset for analysis the sentiment like positive, negative and neutral. These classification of sentiment analysis used existing algorithm NB (89.53), SVM (93.5) and the proposed algorithm IGAB algorithm (97.4) is better than other 
algorithm. The novel algorithm produced better result in precision, recall and time. For our future work is trying to implement in internet base application and dynamically retrieve the post and comment from different social media data to be analysis the sentiment. Also added to the future works deal with the additionally added methods and includes the score to improve the efficiency of the performance. The calculation of the score is the new semantic information to the traditional machine learning techniques and it is provide better result of sentimental classification compared with the existing method.

\section{REFERENCES}

[1] B.Pangand L. Lee, "Opinion Mining And Sentiment Analysis," Foundations And Trends In Info Retrieval, Vol.2, No.1-2,Pp. 1-135,2008

[2] J.Bollen, H. Mao, And A. Pepe, "Modeling Public Mood And Emo-Tion: Twitter Sentiment And SocioEconomic Phenomena," Inproc. 5th Int. Aaai Conf. Weblogs Social Media, Barcelona, Spain, 2011.

[3] G. Heinrich, "Parameter Estimation For Text Analysis," Fraunhofer Igd, Darmstadt, Germany, Univ. Leipzig, Leipzig, Germany, Tech. Rep., 2009.

[4] H. Becker, M. Naaman, And L. Gravano, "Learning Similarity Metrics For Event Identification In Social Media," In Proc. 3rd Acm Wsdm, Macau, China, 2010.

[5] Paolo Fornacciari, Monica Mordonini, Michele Tomauiolo," Social Network And Sentiment Analysis On Twitter:Towards A Combined Approach".

[6] Rajni Singh, Rajdeep Kaur," Sentiment Analysis On Social Media And Online Review", International Journal Of Computer Applications (0975 - 8887) Volume 121 - No.20, July 2015.
[7] T.-K.Fanandc.-H.Chang, "Sentiment-Oriented Discourse Advertising," Information And Knowledge Systems, Vol.23, No.3,Pp. 321-344,2010

[8] Y.Lu, C.Zhai, And N.Sundaresan, "Rated Side Account Of Short Comments," In Www2009, 2009 , Pp.131-140

[9] Pooja Khanna, Sachin Kumar, Sumita Mishra , Anant Sinha," Sentiment Analysis: An Approach To Opinion Mining From Twitter Data Using R", International Journal Of Advanced Research In Computer Science, Volume 8, No. 8,SeptemberOctober 2017

[10] G. Parthasarathy, D. C. Tomar," A Survey Of Sentiment Analysis For Journal Citation", Indian Journal Of Science And Technology, Vol 8(35), Doi: 10.17485/Ijst/2015/V8i35/55134, December 2015, Issn (Print) : 0974-6846, Issn (Online) : 0974-5645.

[11] Surya Prakash Sharma, Dr Rajdev Tiwari, Dr Rajesh Prasad," Opinion Mining And Sentiment Analysis On Customer Review Documents- A Survey", International Conference On Advances In Computational Techniques And Research Practices Noida Institute Of Engineering \& Technology, Greater Noida, Vol. 6, Special Issue 2, February 2017

[12] Akshi Kumar, Teeja Mary Sebastian," Sentiment Analysis On Twitter", Ijcsi International Journal Of Computer Science Issues, Vol. 9, Issue 4, No 3, July 2012, Issn (Online): 1694-0814

[13] Xing Fang, Justin Zhan," Sentiment Analysis Using Product Review Data", Fang And Zhan Journal of Big Data (2015) 2:5 Doi 10.1186/S40537-015-00152.

[14] Brian Keith, Exequiel Fuentes, Claudio Meneses," A Hybrid Approach For Sentiment Analysis Applied To Paper", Kdd'17, August 2017, Halifax, Nova Scotia, Canada. 